\title{
Ultra-flat Nickel substrates for Scanning Probe Microscopy of polyhistidine-tagged proteins
}

\author{
Andrea Alessandrini ${ }^{1,2}$, Carlo Augusto Bortolotti ${ }^{1}$, Giovanni Bertoni ${ }^{3}$, Alessandro Vezzoli $^{3}$ \\ $\&$ Paolo Facci ${ }^{1}$
}

${ }^{1}$ CNR-INFM National Research Center on "Nanostructures and BioSystems at Surfaces - S3", Via Campi 213/A, 41100 Modena, Italy.

${ }^{2}$ Department of Physics, University of Modena and Reggio Emilia, Via G. Campi, 213/A, I-41100, Modena, Italy.

${ }^{3}$ Department of Biomolecular Sciences and Biotechnology, University of Milan, Via Celoria, 26, 20133 Milan, Italy.

\section{Supporting information}

For XPS analysis, a freshly stripped Nickel substrate was inserted in the Ultra-High-Vacuum measurement chamber (base pressure $1 \times 10^{-8} \mathrm{~Pa}$ ) and the measurements were performed using a non-monochromatized X-ray $\mathrm{Mg} \mathrm{K}_{\underline{\alpha}}$ source $(h v=1253.6 \mathrm{eV})$. The energy resolution was $1.9 \mathrm{eV}$ for the wide binding energy survey and $1 \mathrm{eV}$ for the small range spectrum. 


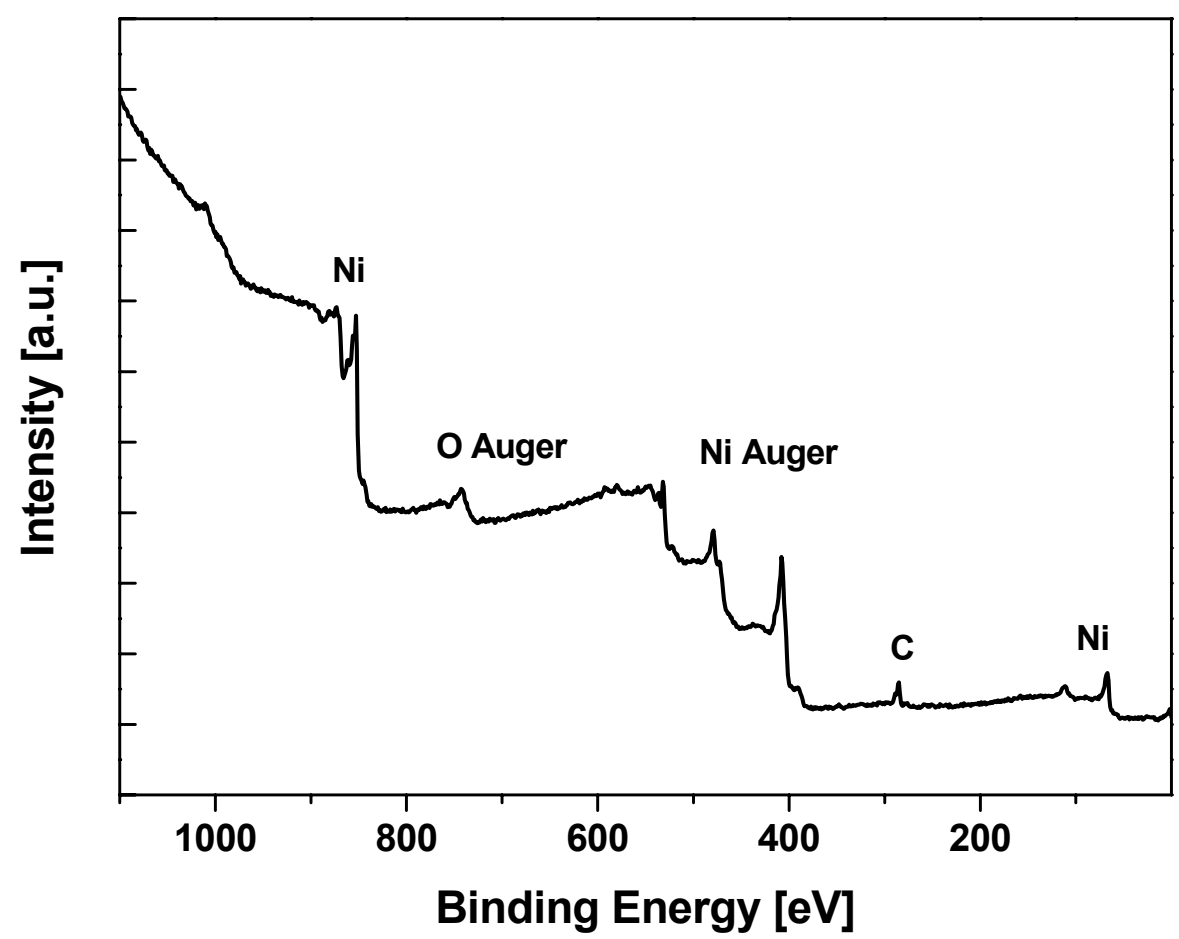

Figure S1: X-ray Photoelectron spectrum of a Nickel substrate obtained immediately after the mica stripping. The wide binding energy range survey has been obtained at a resolution of $1.9 \mathrm{eV}$ with a pass energy of $200 \mathrm{eV}$. The spectrum shows mainly the presence of Nickel, Oxygen and Carbon. The last two elements are probably due to environmental contamination.

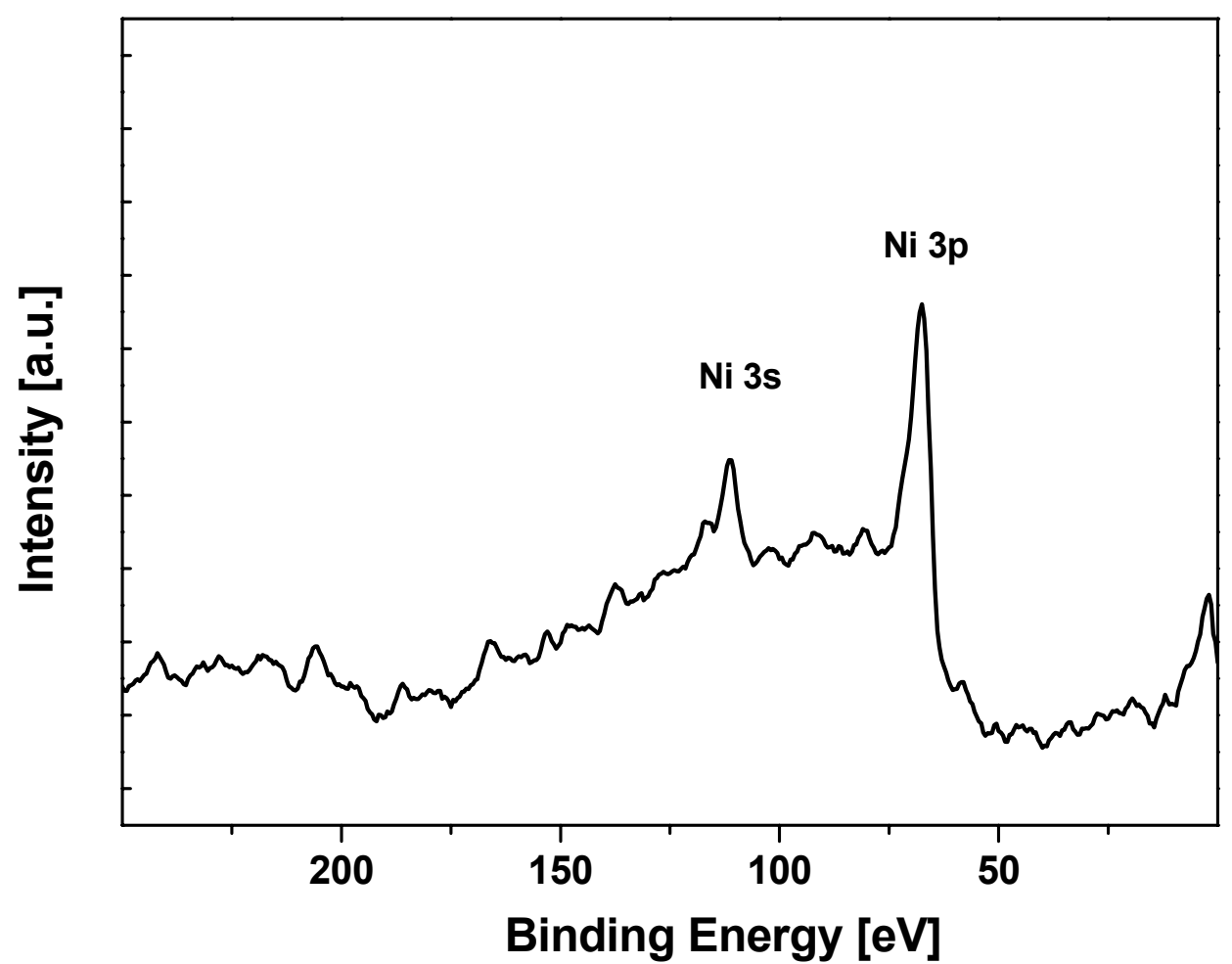

Figure S2: Small range X-Ray Photoelectron spectrum of the same sample of figure S1. The spectrum has been obtained at a resolution of $1 \mathrm{eV}$. The two peaks in the spectrum are due to Nickel whereas no evidence of $\mathrm{Al}$ (peak $\sim 74 \mathrm{eV}$ ) or $\mathrm{Si}$ (peaks $\sim 100 \mathrm{eV}$ and $\sim 150 \mathrm{eV}$ ) from the mica appears. 